\title{
Performance of the first ALMA Band 5 production cartridge.
}

\author{
Bhushan Billade, Student Member, IEEE, Olle Nyström, Student Member, IEEE, Denis Meledin, Erik Sundin, \\ Igor Lapkin, Mathias Fredrixon, Vincent Desmaris, Member, IEEE, Hawal Rashid, Magnus Strandberg, Sven-Erik \\ Ferm, Alexey Pavolotsky and Victor Belitsky, Senior Member, IEEE.
}

\begin{abstract}
We present performance of the first ALMA Band 5 production cartridge, covering frequencies from $163 \mathrm{GHz}$ to 211 GHz. ALMA Band 5 is a dual polarization, sideband separation (2SB) receiver based on all Niobium $(\mathrm{Nb})$ SuperconductorInsulator-Superconductor (SIS) tunnel junction mixers, providing $16 \mathbf{G H z}$ of instantaneous RF bandwidth for astronomy observations. The 2SB mixer for each polarization employs a quadrature configuration. The sideband separation occurs at the output of the IF hybrid that has integrated bias-T for biasing the mixers, and is produced using superconducting thin film technology.

Experimental verification of the Band 5 cold cartridge performed together with warm cartridge assembly, confirms that the system noise temperature is below $45 \mathrm{~K}$ over most of the $\mathrm{RF}$ band, which is less than five photon noise $(5 \mathrm{hf} / \mathrm{k})$. This is to our knowledge, the best results reported at these frequencies. The measurement of the sideband rejection indicates that the sideband rejection better than $10 \mathrm{~dB}$ over $90 \%$ of the observational band.
\end{abstract}

Index Terms-Terahertz System, Astronomy instruments, ALMA, Superconducting devices, Millimeter wave mixers, Superconductor-insulator-superconductor mixers, Thin film circuits.

\section{INTRODUCTION}

$\mathbf{T}$ HE Atacama Large Millimeter/sub-millimeter Array (ALMA) is a radio interferometer under construction by an international consortium consisting of European countries (ESO), USA, Canada, Chile, Taiwan and Japan. ALMA is located at 5000 meters above sea level in the Atacama Desert in Chile, where the earth's atmosphere provide the most favorable conditions for radio astronomy observations at these frequencies. ALMA will cover the frequencies from $31 \mathrm{GHz}$ to $950 \mathrm{GHz}$ split into ten frequency bands. With its more than 60 antennas of $12 \mathrm{~m}$ diameter and a reconfigurable baseline ranging from $150 \mathrm{~m}$ to $18 \mathrm{Km}$, ALMA will offer unprecedented sensitivity and resolution.

The work presented here concerns the design and development of the ALMA Band 5 receiver. ALMA Band 5 is funded by the European Commission's sixth Framework Program (FP6), an infrastructure enhancement project. In this framework program, the project will supply 6 receiver cartridges

Manuscript received on August 01, 2011. This work was supported by EC Framework Program 6 (FP6), under infrastructure enhancement contract 515906

Bhushan Billade, Olle Nyström, Denis Meledin, Erik Sundin, Igor Lapkin, Mathias Fredrixon, Vincent Desmaris, Hawal Rashid, Magnus Strandberg, Sven-Erik Ferm, Alexey Pavolotsky and Victor Belitsky are with the Group of Advanced Receiver Development (GARD) at Chalmers University of Technology, Gothenburg, Sweden. (phone: +46 31772 1851, e-mail: bhushan.billade@chalmers.se). to the ALMA project for integration into the ALMA frontend receiver. Similar to other ALMA bands, the Band 5 receiver is also divided into two separate units, a warm cartridge assembly (WCA) and a cold cartridge assembly (CCA).

The Group for Advanced Receiver Development at Chalmers University with Onsala Space Observatory is responsible for the design and development of the cold cartridge assembly (CCA) and the STFC Rutherford Appleton Laboratory, UK, is responsible for the design and development of the Band 5 warm cartridge assembly (WCA) and the local oscillator (LO) chain.

The CCA is a unit which is cryogenically cooled using a three stage cryo-cooler of the ALMA front end cryostat. Different components of the cartridge are thermally connected to different temperature stages of the cooler. The cold cartridge assembly hosts, receiver optics, orthomode transducer (OMT), SIS mixers, IF hybrid, IF low noise amplifiers, mixer bias and ESD protection circuitry and $\mathrm{x} 6$ multiplier which is delivered by RAL.

The warm cartridge assembly is a unit which resides outside the cryo-cooler and provides a blind mate interface to the cold cartridge assembly. The warm cartridge assembly hosts the local oscillator source operating from $14 \mathrm{GHz}$ to $17 \mathrm{GHz}$, a $\mathrm{x} 2$ multiplier, phase lock loop for LO after the $\mathrm{x} 2$ multiplier stage, warm IF amplifiers and bias and control circuitry.

\section{ALMA BAND 5 COLD CARTRIDGE}

The ALMA Band 5 receiver is a dual polarization, sideband separating, heterodyne receiver, covering frequencies from 163 $\mathrm{GHz}$ to $211 \mathrm{GHz}$, with 4-8 GHz down converted intermediate frequency (IF) for each channel. Band 5 receiver employs sideband separation quadrature configuration (2SB) based on all Niobium (Nb) Superconductor-Insulator-Superconductor (SIS) mixers [1]. The separation of two orthogonal polarizations is realized using a waveguide orthomode transducer [2]. For each polarization branch, the receiver will provide $8 \mathrm{GHz}$ instantaneous RF band for observations. Among the other frequency bands of the ALMA project, Band 5 is the lowest frequency band that uses all cold optics. Consequently, the physical dimensions for all the optics components for Band 5 are largest compared to all other ALMA bands. The optics components packed inside a limited space of $\emptyset 170 \mathrm{~mm}$, leave very little room for the other receiver components.

Fig. 1 shows a CAD drawing of the cold cartridge, with the fiberglass supports removed for better visibility. It can be seen 


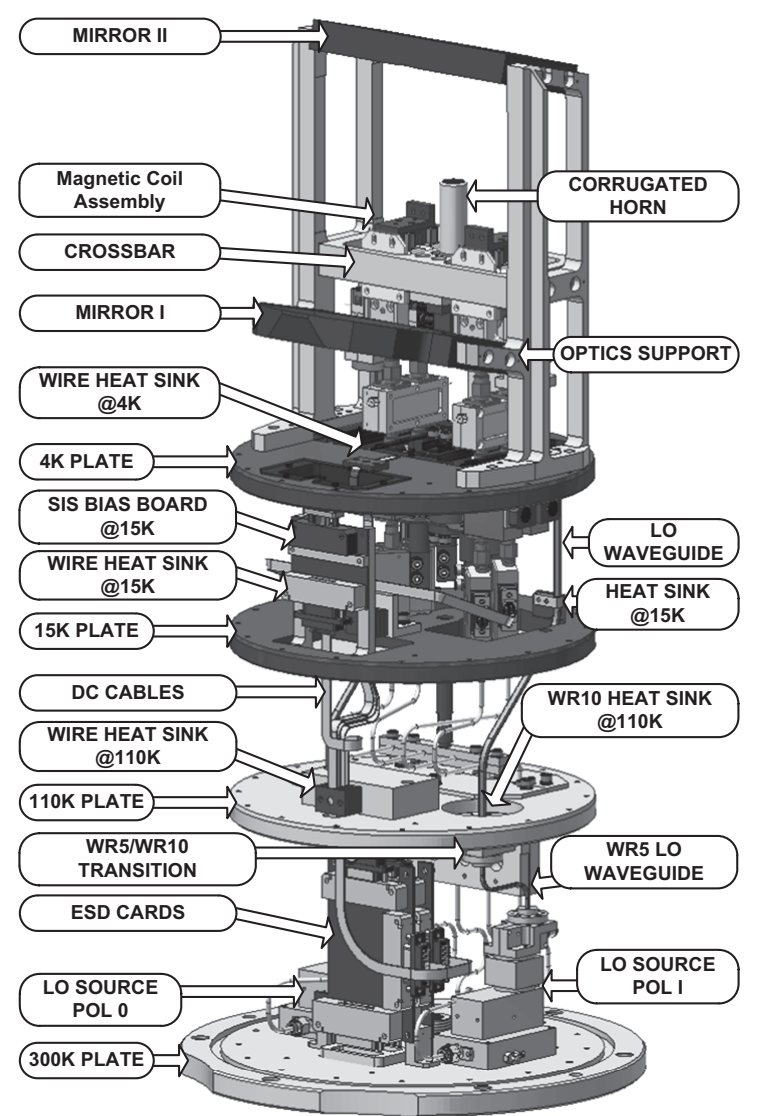

Fig. 1. A CAD drawing of the entire cold cartridge assembly (CCA), showing different temperature stages and arrangement of receiver components inside the cartridge envelop, the fiberglass supports separating different temperature stages are removed for visibility of components inside.

from the figure that the mirrors along with the optics supports occupy much of the space on the $4 \mathrm{~K}$ stage. The design parameters of Band 5 mirrors and corrugated horn are based on the design proposed by M. Carter et al., [3] and has been verified using physical optics simulation by $\mathrm{M}$. Whale et al., [4]. The optics dimensions put strong constraints on the sizes of all the receiver components and demand a very compact design. A mixer block design with waveguide back piece layout [1], [5], [6] allows very compact design of the mixer block and also the IF output pointing in desirable direction. Furthermore, to avoid extra cables, all the components in the chain are directly attached to each other with SMA connectors. Keeping compactness of all the components in mind and in order to take advantage of cold temperature, we chose a custom made superconducting IF hybrid that fits the distance between the SMA connectors of the 2SB mixer IF outputs avoiding any unnecessary cabling. Apart from tight constraints on the size of all the receiver components, we have very limited cooling capacity at the $4 \mathrm{~K}$ stage, restricting the total power dissipation at the $4 \mathrm{~K}$ stage to merely $36 \mathrm{~mW}$. These 36 $\mathrm{mW}$ are shared between the four SIS mixers, magnetic coils, low noise amplifiers and thermal load due to heat conduction. A lot of effort has been put to reduce the contribution from each of these components but still it does not allow us to integrate the DC bias circuit for the SIS mixers at the $4 \mathrm{~K}$ stage [7]. Therefore, in our design, as shown in Fig. 1, the DC biasing to the mixer is done using a bias circuitry placed at the $15 \mathrm{~K}$ plate and integrating a bias-T with the IF hybrid; the DC biasing is thus achieved through the output SMA connector of the mixers. The hybrid is followed by a $4-8 \mathrm{GHz}$ isolator and a cryogenic HEMT low noise amplifier.

Part of the local oscillator chain resides inside the cold cartridge assembly and is placed on the $300 \mathrm{~K}$ plate of the cartridge. The LO chain placed at $300 \mathrm{~K}$ stage includes a x3 active multiplier from QuinStar, followed by an amplifier and isolator both produced by RPG GmbH and a x 2 doubler developed by RAL [8], [9]. The LO signal is then guided from the $300 \mathrm{~K}$ stage to $4 \mathrm{~K}$ stage to the mixers using overmoded (WR10) waveguide. In order to reduce the thermal coupling between the different temperature stages, we use stainless steel waveguide with heat sinks at all the temperature stages.

\section{BAND 5 COLD CARTRIDGE KEY COMPONENTS}

\section{A. Mixer assembly}

The 2SB mixer employs a modular design approach to facilitate characterization of each component separately. Fig. 2 shows an assembly comprising of a corrugated horn, orthomode transducer (OMT) and the mixer assembly. The $90^{\circ}$ waveguide twist after the OMT is used so that both polarizations have the same orientation.

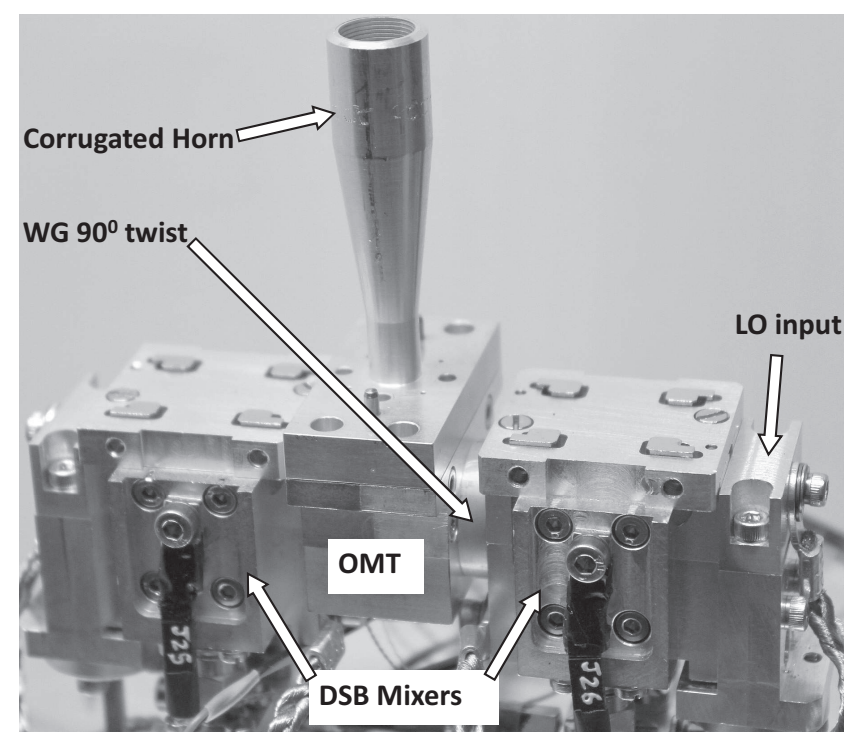

Fig. 2. A picture of the cartridge showing, the horn, OMT, 2SB mixers and the $90^{\circ}$ waveguide twist. Coils, mirrors and optical support structures are removed for better visibility.

The sideband separation mixer [1] uses end piece configuration, with identical back pieces for both DSB mixers while the mixer chips in the two DSB back pieces are mirrored copy of each other. Fig. 3 shows the picture of the 2SB mixer. The middle piece houses an in-phase LO splitter [10] and $90^{\circ} \mathrm{RF}$ hybrid. All the mechanical components of the 2SB mixer are machined in tellurium copper and electroplated with gold.

The RF hybrid is an 8-section waveguide branch line coupler designed to achieve broadband performance [11], 


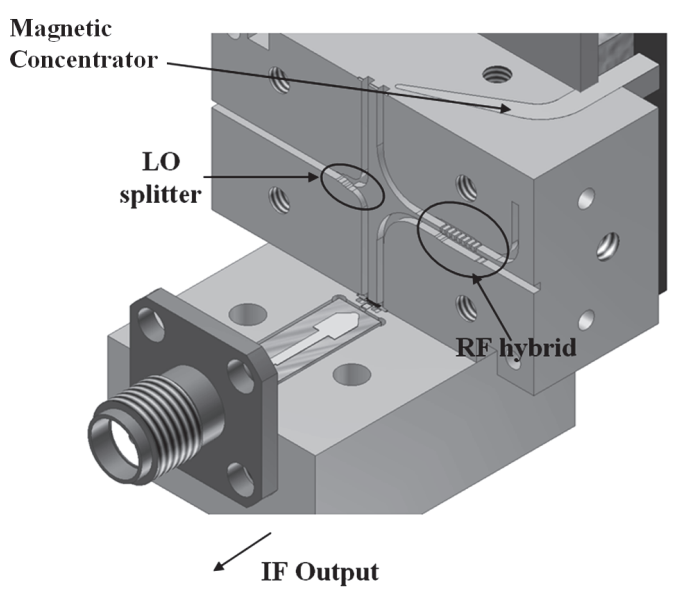

Fig. 3. Picture of middle piece, with RF hybrid and LO splitter.

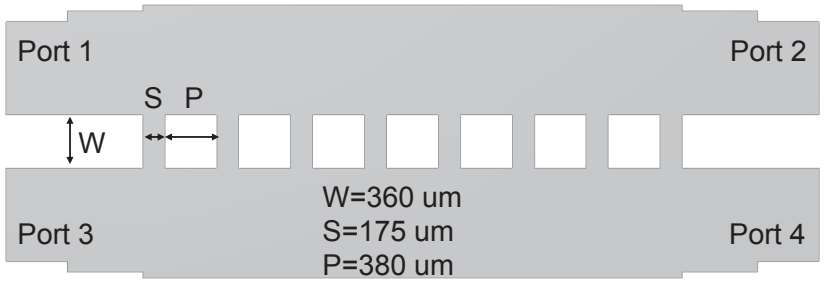

Fig. 4. Layout of the RF Hybrid, the waveguide dimensions are $1260 \mu \mathrm{m}$ $630 \mu \mathrm{m}$.

[12]. The RF hybrid is designed and simulated using CST microwave studio and Agilent EMDS. Fig. 4 shows the layout of the RF hybrid. The simulation results in Fig. 5 indicate that the maximum amplitude imbalance of $0.8 d B$ can be achieved across the whole RF band, with negligible phase imbalance.

For the SIS mixer, we employ a MMIC-like approach where most of the DSB mixer components along with the LO coupler are integrated on the same z-cut crystal quartz substrate. The dimensions of the on-chip LO coupler are defined by photolithography, providing greater precision over the definition of coupler geometry and thus allowing accurate control over the LO coupling.

As shown in Fig. 6 an E-plane probe is used for the waveguide to microstrip transition for both RF and LO signal, a RF choke structure provides virtual RF ground for the $\mathrm{RF}$ and LO signals in microstrip mode. The choke is also $\mathrm{DC} / \mathrm{IF}$ grounded to the chassis using bond wires. The LO circuitry includes a probe and an impedance transformer to bring the probe impedance to a desirable value. LO injection is implemented using a microstrip line coupler with slots in the ground plane. The isolated port of the coupler is terminated using a wideband elliptical floating load [13]. The down converted signal at intermediate frequency (IF) is extracted between the RF and LO waveguide.

At intermediate frequencies, the RF and LO matching components, including the transmission lines and the LO coupler structure appears as a capacitor and hence affect the mixer IF performance, especially at the higher end of the IF band. It is therefore necessary that the whole RF and LO circuitry
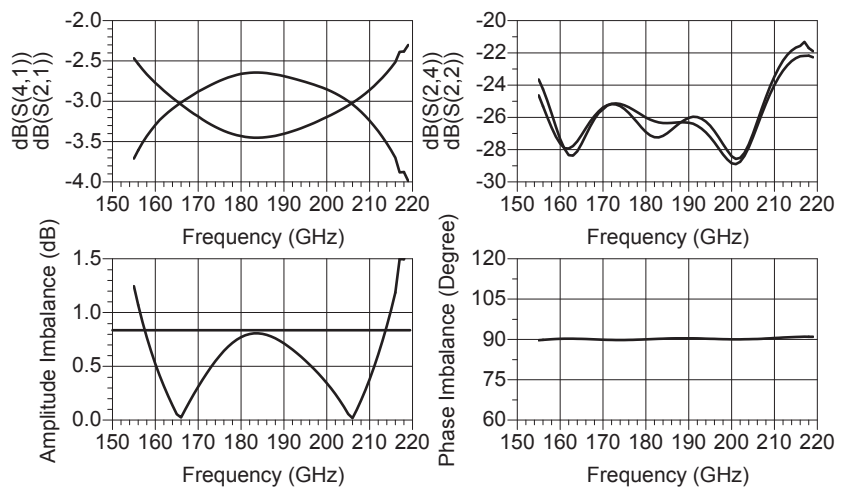

Fig. 5. Simulation of the RF hybrid, top left plot shows the output at through and coupled port, bottom left plot shows the amplitude imbalance, and the dotted line represents $0.85 \mathrm{~dB}$. Top right plot shows the isolated port and reflection at one of the port (reflection at other three ports not shown but similar), bottom right plot shows the phase imbalance.

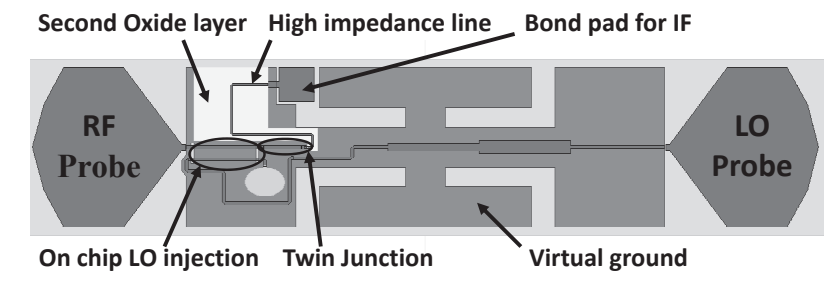

Fig. 6. ALMA Band 5 mixer chip layout showing the RF choke structure, RF and LO probe, LO coupler, RF and LO matching circuitry, on chip resistive termination and SIS junctions.

represents as low capacitance as possible at the IF frequencies. At the same time it is very important that the LO coupler has minimal losses at RF frequencies. Therefore, the LO coupler has been designed to have very low coupling of $-18 \mathrm{~dB}$, this ensures that the LO circuitry does not introduce any losses at $\mathrm{RF}$ frequencies, and we chose a coupler design with slots in the ground plane, which ensures that the coupler contribute very little to the overall IF capacitance of the chip.

\section{B. IF Hybrid}

In order to avoid unnecessary IF cabling inside the Band 5 cartridge, all the components are directly connected to each other via SMA connectors. For this reason, any commercially available IF hybrid cannot be used, and hence the IF hybrid was specially designed and built. The pitch between the two SMA connectors of 2SB mixer defines the distance between the two input SMA connectors of the hybrid. The IF hybrid is fabricated on 500 micron thick Alumina substrate and glued to the gold plated copper housing using a silver conductive epoxy.

The IF hybrid is designed for frequency 4-8 GHz using Lange coupler layout, and to take advantage of the cryogenic temperatures the IF hybrid is fabricated with superconducting $\mathrm{Nb}$ lines to eliminate conductive losses. A thin Palladium layer is deposited onto the Niobium to allow bonding where it is necessary.

In order to avoid substrate modes, which could compromise the hybrid performance, the complete IF hybrid is divided into 


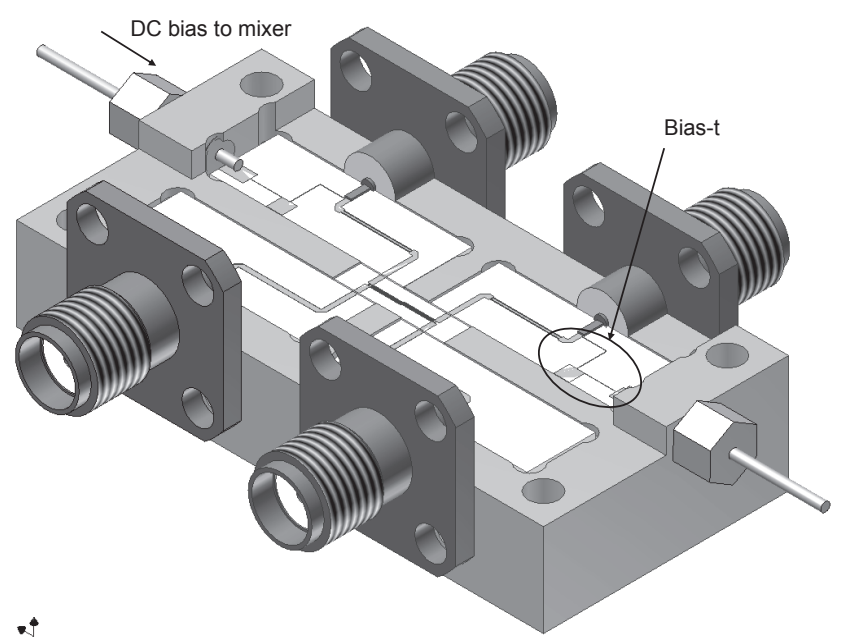

(a)

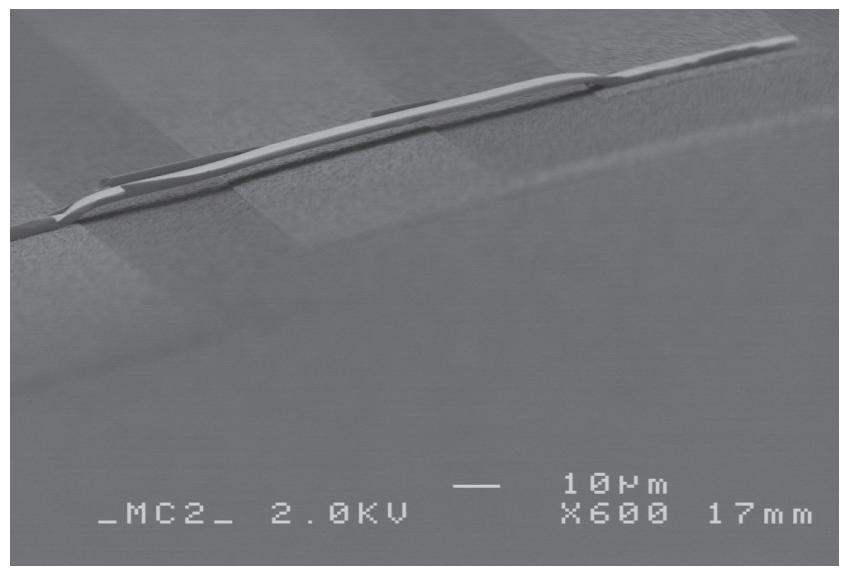

(b)

Fig. 7. IF hybrid with integrated bias-T (a) Layout of the hybrid with capacitive feed-through for DC bias, (b) SEM image of the air bridge fabricated along with the Lange coupler.

five separate sections, one for the Lange coupler, two for $50 \Omega$ connecting line and two for the bias-T. The lines, bias-T and the Lange coupler are connected using paired bond wires to reduce parasitic inductance of the inter-connection.

Fig. 7 (a) shows the layout of the IF hybrid. The width of the coupled lines of the Lange coupler is $45 \mu \mathrm{m}$ with spacing between the lines $34 \mu \mathrm{m}$. The Lange coupler uses air bridges fabricated together with the hybrid using thin film technology. Fig. 7 (b) shows the scanning electron microscope image of the air bridge that connects the fingers of the Lange coupler, the measured height of the air bridge is $3 \mu \mathrm{m}$.

Fig. 8 shows the simulation result with 3 micron height of the air-bridge, the maximum amplitude variation is $0.7 \mathrm{~dB}$ and phase variation is negligible.

\section{IF Amplifiers}

The cold IF amplifier used for ALMA Band 5 is based on previously developed 3-stage HEMT amplifier for the Swedish Heterodyne Facility Instrument for the APEX telescope [14]. In contrast to the earlier design, the amplifier uses HRL InP transistors for the first stage (provided by ESO for the
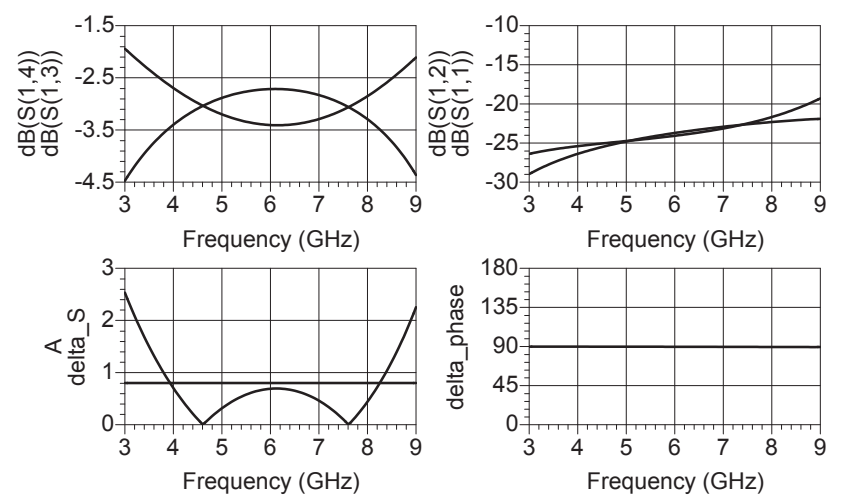

Fig. 8. Simulation result of the IF hybrid, top left plot shows the output at through and coupled port, bottom left plot shows the amplitude imbalance, and the dotted line represents $0.6 \mathrm{~dB}$. Top right plot shows the isolated port and reflection at one of the port (reflection at other three ports not shown but similar), bottom right plot shows the phase imbalance.

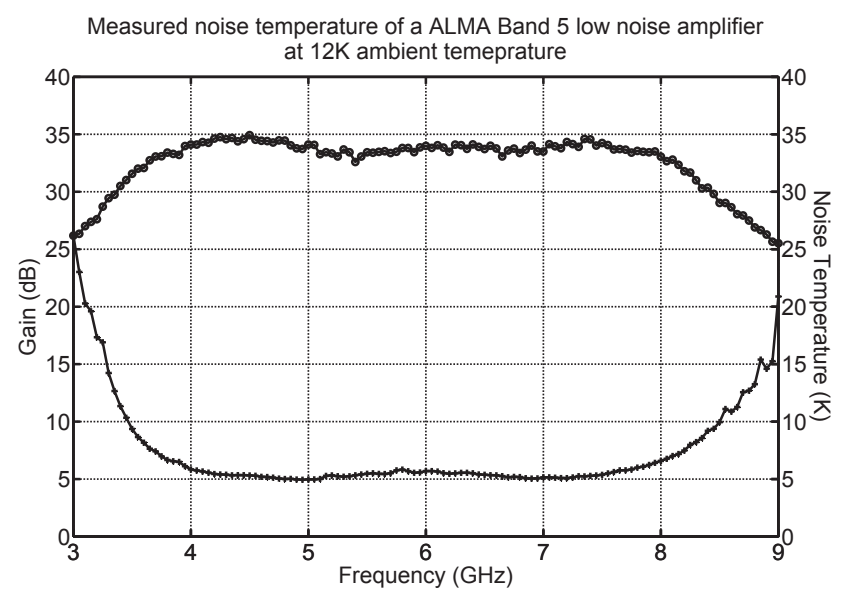

Fig. 9. Measurement of one of the ALMA Band 5 low noise amplifiers performed at $12 \mathrm{~K}$ ambient temperature, showing $35 \mathrm{~dB}$ gain and noise temperature below $6 \mathrm{~K}$. Measurements of all other amplifiers shows consistent performance.

ALMA Band 5 project) and for the subsequent two stages uses Mitsubishi MGFC4419G InGaAs pseudomorphic HEMT. Fig. 9 shows the measured performance for one of the Band 5 low noise amplifier. The amplifier has $35 \mathrm{~dB}$ gain over the 4-8 $\mathrm{GHz}$ band with noise temperature less than $6 \mathrm{~K}$ over the entire band, while consuming merely $6 \mathrm{~mW}$ of power.

\section{Measurement Results}

\section{A. Noise temperature measurement}

The performance verification of the Band 5 cold cartridge was carried out together with the warm cartridge assembly delivered by the Rutherford Appleton Laboratory, UK. To perform these tests we used the NAOJ cartridge test cryostat [15]. Most of the measurements are done using an automated system, built around the test cryostat [16].

The noise specifications for the ALMA Band 5 project requires the system noise temperature to be below $65 \mathrm{~K}$ over $80 \%$ of the band and less than $108 \mathrm{~K}$ at any frequency. Fig. 10 shows the measured noise performance of the first Band 5 production cartridge. The noise was measured over the 4-8 


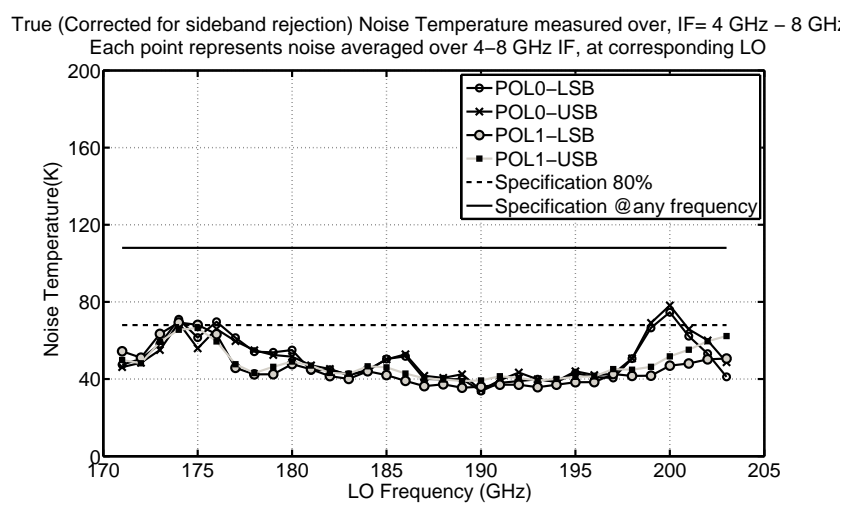

Fig. 10. Measurement of noise temperature for ALMA Band 5 CCA 01, for both sidebands and polarization, measured over the $4-8 \mathrm{GHz}$ IF band.

GHz IF band for both sidebands and polarizations and includes the contribution from the dewar windows, IR filters and takes into account all the noise contributions up to the IF output ports of warm cartridge assembly. The noise measurements were carried out using standard Y-factor method with liquid nitrogen and room temperature loads. The presented noise temperature also takes into account the correction for the sideband rejection [17]. Our measurements confirm that the receiver performance meets all the noise specification for the Band 5 project and is in most cases better than the specifications with a good margin.

\section{B. Sideband rejection measurement}

In sideband separation millimeter wave receiver, knowledge of image rejection is very important. Unless the image rejection is very high, a correction term is required for the estimation of the system noise temperature. We use the technique proposed by A. Kerr [17] to estimate the sideband rejection. Fig. 11 (a) shows the sideband rejection measurement results, performed at RF frequencies from 163 to $211 \mathrm{GHz}$ with 100 $\mathrm{MHz}$ frequency steps and Fig. 11 (b) shows the same measurements plotted across the IF band for all RF frequencies. The measurements confirm that the sideband rejection is better than $10 \mathrm{~dB}$ over $90 \%$ of the band and better than $7 \mathrm{~dB}$ over $99 \%$.

\section{Receiver stability}

1) Amplitude stability: One of the most important design parameter for a modern radio telescope is the stability of the instrument. The stability of the receiver is generally described in terms of its Allan variance time $\left(T_{A}\right)$. The Allan variance time describes the integration time for a receiver beyond which the observing efficiency is reduced. Fig. 12 shows the receiver stability measurements using the Allan variance method. Measurements were performed at 3 different LO frequencies and total output power over 4-8 GHz IF band is used to calculate the Alan variance. It is evident from the Fig. 12 that the receiver meets the specification with margin for all the measured frequencies.

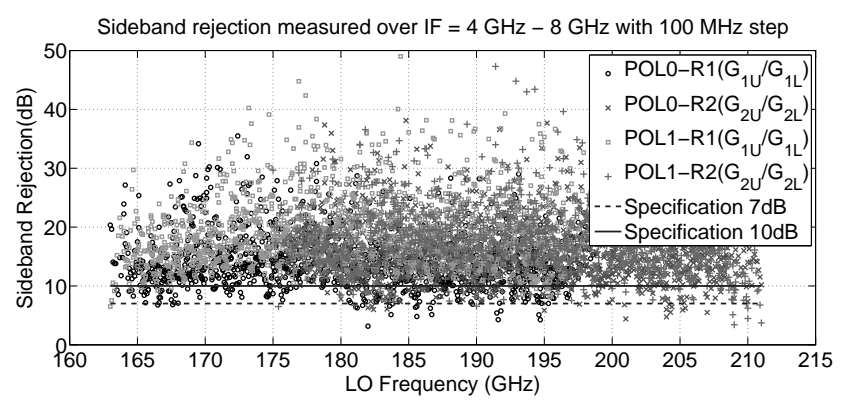

(a)

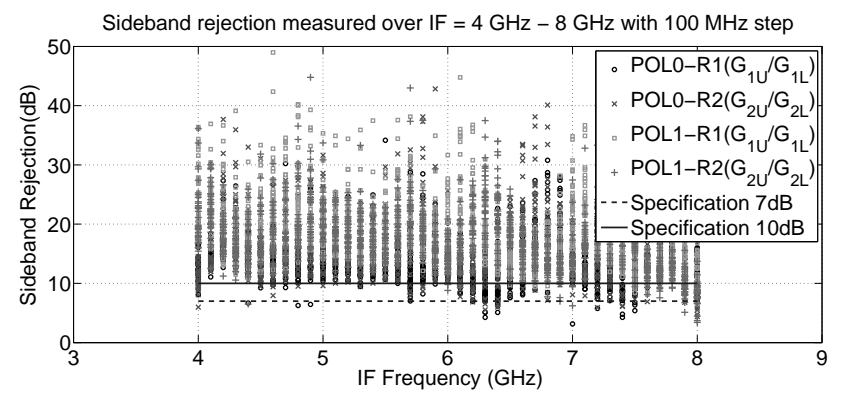

(b)

Fig. 11. Measurement of sideband rejection/image rejection for ALMA Band 5 CCA 01, for both polarization, (a) Sideband rejection vs. RF (b) Sideband rejection vs. IF.

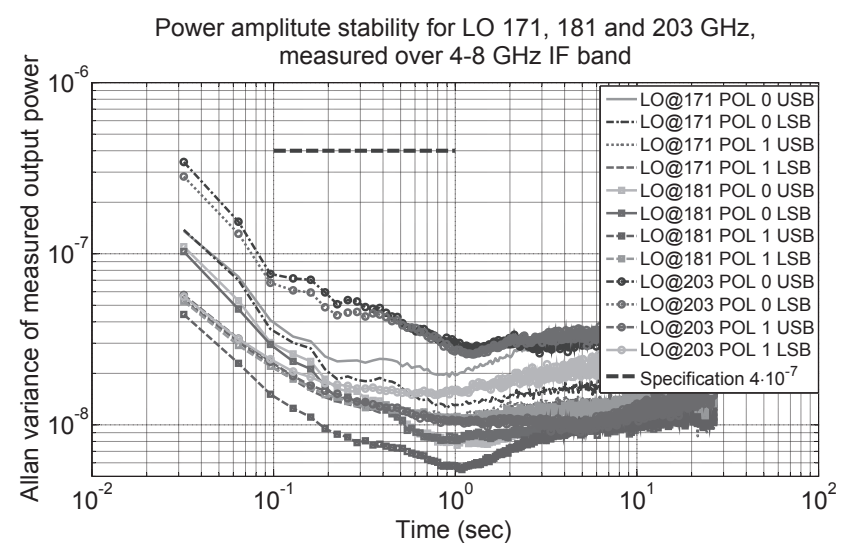

Fig. 12. Measurement of receiver stability using the Allan variance method, the total IF power is measured over the $4-8 \mathrm{GHz}$ bandwidth.

2) Signal path phase stability: Along with signal amplitude stability, for a radio interferometer it is equally important to have very good phase stability of the signal path. To ensure a stable baseline, ALMA Band 5 project specification requires that for all frequencies within the IF pass-band the signal path transfer function should maintain phase stability better than 0.9 degrees on timescales up to 300 seconds. The phase being the average value measured in $16 \mathrm{msec}$. The signal path includes all components between the RF window and the IF outputs of the warm cartridge assembly that houses the second-stage amplifier and the local oscillator chain. The required phase stability excludes any contribution from the local oscillator chain residing in the WCA but takes into account contributions from the LO components inside the CCA. Fig. 13 shows the measured phase stability of the signal 


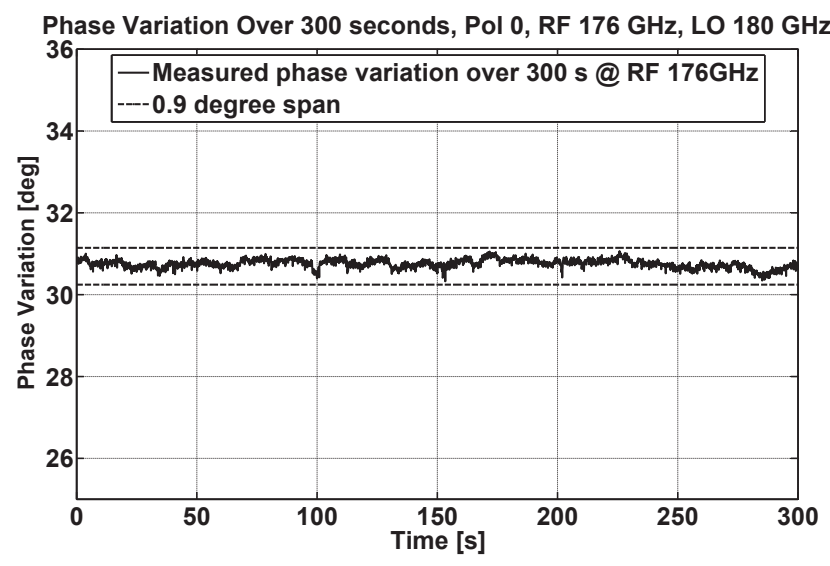

Fig. 13. Measurement of signal path phase stability, measured at $180 \mathrm{GHz}$ LO frequency and $176 \mathrm{GHz}$ RF frequency. Plot shows $300 \mathrm{~s}$ measurement data of the phase of $4 \mathrm{GHz}$ down converted IF at the lower sideband. The two dotted lines shows 0.9 degree span.

path. For the measurement of phase stability, a LO signal at $180 \mathrm{GHz}$ and a RF signal at $176 \mathrm{GHz}$ were used and the phase of the down-converted IF was measured at $4 \mathrm{GHz}$ IF at the lower sideband. Fig. 13 shows the measurement results of the phase stability confirming signal path phase stability less than 0.9 degrees over time period of $300 \mathrm{~s}$.

\section{CONCLUSION}

We have designed and built the first ALMA Band 5 production cartridge and fully characterized it. The characterization of the cold cartridge was performed with integrated warm cartridge assembly. Performance verification of the entire system including cryostat window, IF filters and all IF components, confirms that the first Band 5 production cartridge meets all ALMA project specifications.

The measurement of 2SB configuration confirms that the system noise temperature less than $45 \mathrm{~K}$ over most of the RF band, which is less than five quantum noise $(5 \mathrm{hf} / \mathrm{K})$ and less than $65 \mathrm{~K}(7 \mathrm{hf} / \mathrm{K})$ over the entire $\mathrm{RF}$ band. This is to our knowledge the best results so far at these frequencies. The sideband separation for both polarizations is better than 10 $\mathrm{dB}$ over $90 \%$ of the band and better than $7 \mathrm{~dB}$ over $99 \%$ of the RF band.

The first ALMA Band 5 cartridge has been delivered to the European ALMA Integration Center at RAL in UK and is expected to be in operation at the ALMA cite in Chile during year 2012 .

\section{ACKNOWLEDGMENT}

This project is funded by EC Framework Program 6 (FP6), under infrastructure enhancement contract 515906.

The author would like to thank Dr. Jacob Kooi (Caltech, USA), Doug Henke (currently at HIA, Victoria, Canada), Richardo Finger (DAS, University of Chile, Santiago) and Dr. Raquel Monje (Caltech, USA) for their contribution at earlier stages of the project.

The author would like to acknowledge the Band 5 warm cartridge team at RAL, for their help and support. Special thanks to Dr. Hui Wang and Prof. Brian Ellison. We would also like to thank Monica Obrocka and Hui Xu.

The author would also like to thank Gert Johnsen and Dr. Dimitar Dochev.

\section{REFERENCES}

[1] B. Billade, V. Belitsky, A. Pavolotsky, I. Lapkin, and J. Kooi, "ALMA band 5 (163-211 GHz) sideband separation mixer," in 20th International Symposium on Space Terahertz Technology, April 2009, pp. 19-23.

[2] S. Asayama and M. Kamikura, "Development of double-ridged waveguide orthomode transducer for the $2 \mathrm{~mm}$ band," Journal of Infrared, Millimeter and Terahertz Waves, vol. 30, no. 6, pp. 573-579, June 2009.

[3] M. Carter et al., "ALMA front-end optics design report," available from ALMA project documentation server, Tech. Rep. FEND-40.02.00.00035-B-REP.

[4] M. Whale, N. Trappe, and V. Belitsky, "Physical optics analysis of the ALMA band 5 front end optics," in Proceedings of the 19th International Symposium on Space Terahertz Technology, Groningen, April 2008, pp. 368-372.

[5] B. Billade, "Design of dual polarisation sideband separation mixer for ALMA band 5," Licentiate Thesis, Chalmers University of Technology, Gothenburg, Sweden, September 2009.

[6] R. Monje, V. Belitsky, C. Risacher, V. Vassilev, and A. Pavolotsky, "SIS mixer for 385-500 GHz with on chip LO injection," in Proceedings of the 18th International Symposium on Space Terahertz Technology, USA, March 2007, pp. 44-49.

[7] M. Strandberg, "Analysis, simulation and cryogenic/mechanical design for ALMA band 5 cartridge," Licentiate Thesis, Chalmers University of Technology, Gothenburg, Sweden, June 2011.

[8] M. Henry, H. Wang, B. Ellison, B. Alderman, P. Huggard, B. Billade, A. Pavolotsky, and V. Belitsky, "Oscillator system development for the ALMA band 5 receiver," in Proceedings of the 4th UK/Europe-China Conference on Millimetre Waves and Terahertz Technologies, Glasgow, UK, Septemper 2011.

[9] B. Thomas, J. Treuttel, B. Alderman, D. Matheson, and T. Narhi, "Application of substrate transfer to a $190 \mathrm{GHz}$ frequency doubler and $380 \mathrm{GHz}$ sub-harmomic mixer using MMIC foundry schottky diodes," in Proceedings of the SPIE Astronomical Instrumentation conference, vol. 7020, Marseille, France, June 2008.

[10] A. Kerr and N. Horner, "A broadband in-phase waveguide power divider/combiner," National Radio Astronomy Observatory, Charlottesville, VA 22903, USA, ALMA Memo 325, October 2000.

[11] J. Kooi, "Design of waveguide directional coupler," California Institute of Technology, Pasadena, CA 91125, USA, Private communication with the author.

[12] S. Srikanth and A. Kerr, "Waveguide quadrature hybrids for ALMA receivers," National Radio Astronomy Observatory, Charlottesville, VA 22903, USA, ALMA Memo 343, January 2001.

[13] R. Monje, V. Vassilev, A. Pavolotsky, and V. Belitsky, "High quality micro-strip termination for MMIC and millimeter-wave applications," IEEE MTT-S international microwave symposium, pp. 1827-1830, June 2005 .

[14] E. Sundin, "Development of cryogenic low noise 4 - $8 \mathrm{GHz}$ HEMT amplifier and its advanced characterization," Licentiate Thesis, Chalmers University of Technology, Gothenburg, Sweden, February 2006.

[15] Y. Sekimoto et al., "Cartridge test cryostats for ALMA front end," National Astronomical Observatory of Japan, ALMA Memo 455, April 2003.

[16] O. Nyström et al., "Integrated setup for THz receiver characterization," in Proceedings of the 21st International Symposium on Space Terahertz. Technology, Oxford, UK, March 2010, pp. 4-6.

[17] A. Kerr, S. K. Pan, and J. E. Effland, "Sideband calibration of millimeterwave receiver," ALMA Memo 357, March 2001. 


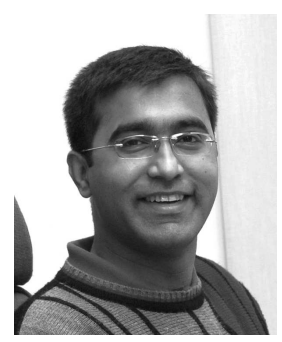

Bhushan Billade (S'10) was born in India, in 1982. He received the B.E. degree in electronics and telecommunication engineering from the $\mathrm{Au}-$ rangabad University, India, in 2003, and the M.Sc. and the Licentiate degree in radio and space science from the Chalmers University of Technology, Gothenburg, Sweden, in 2005 and 2009, respectively, and is currently working towards the Ph.D. at the Department of Earth and Space Science at Chalmers University of Technology.

In 2006, he was with the National Center for Radio Astrophysics, Pune, India. His research interests include microwave and millimeter wave MMIC, heterodyne receivers and mixers. His main focus is on low noise instrumentation for radio astronomy.

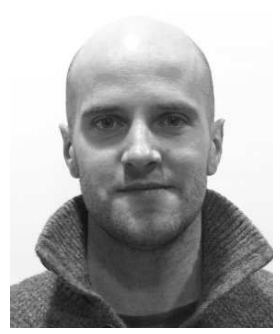

Olle Nyström (S'11) was born in Österbymo, Sweden, in 1979. He received the M. Sc. degree in electrical engineering from Chalmers University of Technology, Gothenburg, Sweden, in 2005 and the $\mathrm{PhD}$ degree in electrical engineering at the same university in 2011.

His research interests are in low-noise THz systems, $\mathrm{THz}$ receiver characterization and calibration, antenna design, and quasi-optics.

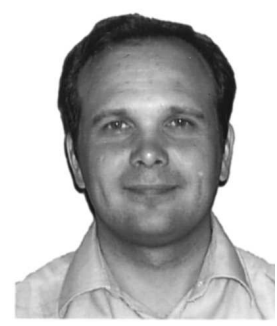

Denis Meledin received the Ph.D. degree in radiophysics from Moscow State Pedagogical University, Russia, in 2003. From 2000 to 2003 he was a predoctoral fellow in the Submillimeter Receiver Lab at the Smithsonian Astrophysical Observatory. Since 2003 he has worked as a research engineer with the Group of Advanced Receiver Development in Chalmers University of Technology, Sweden. His main research activities have been focused on radio astronomy instrumentation.

His current research interests include superconducting low noise heterodyne receivers for $\mathrm{THz}$ applications and the design of microwave and sub-millimeter wave components.

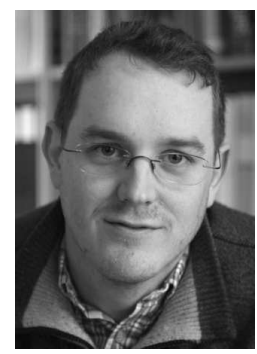

Erik Sundin received the Licentiate degree from Chalmers University of Technology, Gothenburg, Sweden, in 2006. Since then he is with the Group for Advanced Receiver Development at Chalmers as Research Engineer. His professional interest is mainly in cryogenic low-noise amplifiers, as well as in measurement hardware and software.

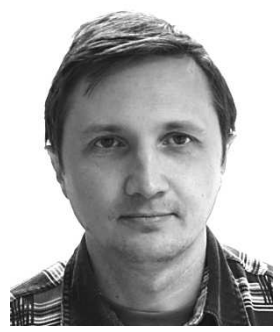

Igor Lapkin received the M.Sc. degree from the State Technical University, Nizhny Novgorod, Russia, in 1985. Currently he is part time research engineer at the Group for Advanced Receiver Development (GARD) at the Department of Earth and Space Sciences, Chalmers University of Technology, Gothenburg, Sweden, and researcher at the Radioreceiving equipment and millimeter-wave radioastronomy department at the Institute of Applied Physics RAS, Nizhny Novgorod.

His current interests are designing and developing heterodyne receivers for radio astronomy and cryogenic techniques.

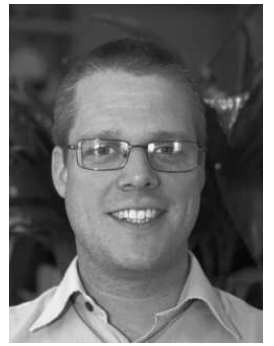

Mathias Fredrixon started working as an engineer at the Group for Advanced Receiver Development (GARD) in 1998. He received his B.Sc. degree in Mechatronics Engineering from Chalmers University of Technology, Gothenburg, Sweden in 2010. $\mathrm{He}$ has been involved in the ODIN project, the Herschel project and the APEX project. Currently he is working as a research engineer for the ALMA B5 project at GARD.

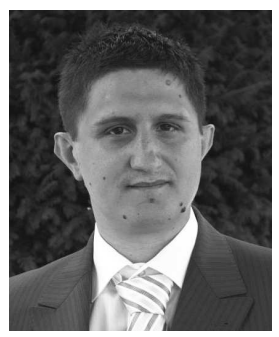

Vincent Desmaris (M'11) received the M.Sc. degree in material science from the National Institute of Applied Science, Lyon, France, in 1999, and the Ph.D. degree in electrical engineering from the Chalmers University of Technology, Gothenburg, Sweden, in 2006. His thesis concerned the fabrication, characterization, and modeling of AlGaN/GaN microwave transistors. Since 2006, he has been with the Group for Advanced Receiver Development (GARD), Chalmers University of Technology, Göteborg, Sweden.

His research interests are in the area of terahertz receiver technology and especially mirofabrication and characterization of waveguide components and circuits as well as planar cryogenic microwave devices.

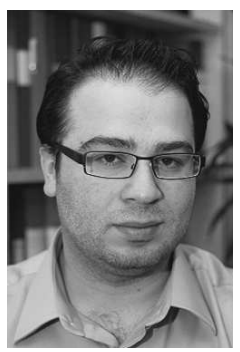

Hawal Rashid was born in Arbil, Iraqi Kurdistan, in 1982. He received the M.Sc. degree in engineering physics from the Chalmers University of Technology, Gothenburg, Sweden, 2010, and working toward $\mathrm{Ph} . \mathrm{D}$.

His research interests are in low-noise and room temperature $\mathrm{THz}$ systems, $\mathrm{THz}$ receiver component design, characterization and calibration.

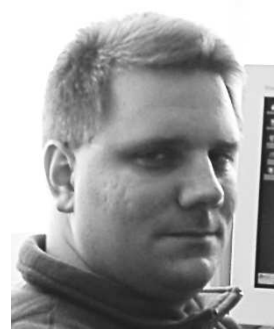

Magnus Strandberg received the B.Sc., M.Sc. in Space Engineering from Umeå University, Sweden, in 2000 and 2002, respectively. In 2002 he joined Group for Advanced Receiver Development (GARD) at Chalmers University of Technology, Gothenburg, Sweden as a Research Engineer. He has been involved in the Herschel project and the APEX project mainly with development of electronics and software development for test system and development/building of control system. He is currently working with the ALMA project where he obtained a technical licentiate from Chalmers University of Technology in 2011 that involved cryogenic and mechanical design of the ALMA Band 5 cartridge.

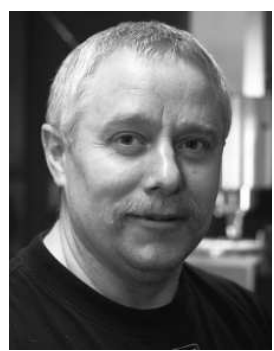

Sven-Erik Ferm was born in Sätila, Sweden, in 1961. From 1980 to 1995 he worked at Hasselblad, Since 1995 he has been working at the department of Earth and Space Science, Chalmers University of Technology, as technician on various projects like ODIN, Herschel, APEX and the ALMA Band 5. 


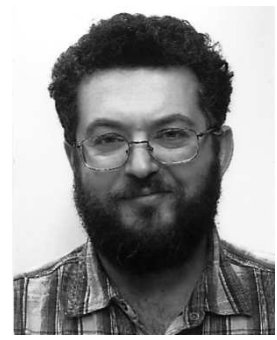

Alexey Pavolotsky received the M.S. and Ph.D. degrees from Moscow Aircraft Technology Institute/Technical University, in 1990 and 2003, both in material science and engineering. Since 2002, he is a Senior Research Engineer in the Group for Advanced Receiver Development, Onsala Space Observatory, Chalmers University of Technology, Gothenburg, Sweden.

His research interests include low-Tc thin film processing and characterization, as well as micro fabrication in general.

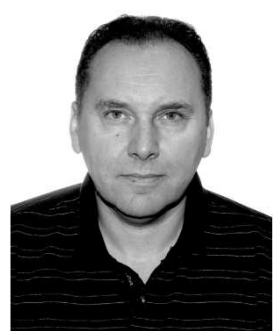

Victor Belitsky (M'95-SM'07) received the M.Sc. degree from the Moscow Telecommunication Institute, Moscow, Russia, in 1977, and the Ph.D. degree in experimental physics from the Institute of Radio Engineering and Electronics, USSR Academy of Sciences, Moscow, Russia, in 1990. He is currently a Professor and leader of the Group for Advanced Receiver Development (GARD) at the Department of Earth and Space Sciences, Chalmers University of Technology, Gothenburg, Sweden.

His research interests include THz electronics and components, instrumentation for radio astronomy and environmental science. 